\title{
Hodge-type integrals on moduli spaces of admissible covers
}

\author{
RENZO CAVALIERI
}

\begin{abstract}
In this paper we study a natural class of intersection numbers on moduli spaces of degree $d$ admissible covers from genus $g$ curves to $\mathbb{P}^{1}$, using techniques of localization. These intersection numbers involve tautological $\lambda$ and $\psi$ classes, and are in some sense analogous to Hodge Integrals on moduli spaces of stable curves.

We compute explicitly these numbers for all genera in degrees 2 and 3 and express the result in generating function form; we provide a conjecture for the general degree $d$ case.
\end{abstract}

$14 \mathrm{C} 30 ; 32 \mathrm{~S} 25,58 \mathrm{~A} 14$

\section{Introduction}

Hodge integrals are a class of intersection numbers on moduli spaces of curves involving the tautological classes $\lambda_{i}$, which are the Chern classes of the Hodge bundle $\mathbb{E}$. In recent years Hodge integrals have shown a great amount of interconnections with Gromov-Witten theory and enumerative geometry.

The classical Hurwitz numbers, counting the numbers of ramified Covers of a curve with an assigned set of ramification data, can be computed via Hodge integrals. Simple Hurwitz numbers have been discussed by Ekedahl, Lando, Shapiro and Vainshtein [4, 5] and by Graber and Vakil [8]; progress towards double Hurwitz numbers has been made by Goulden, Jackson and Vakil [7].

Various spectacular computations of Hodge integrals were carried out in the late nineties by Faber and Pandharipande [6]. Their results have been used to determine the multiple cover contributions in the GW invariants of $\mathbb{P}^{1}$, thus extending the well-known Aspinwall-Morrison formula in Gromov-Witten Theory.

Hodge integrals are also at the heart of the theory developed by Bryan and Pandharipande [3], studying the local Gromov-Witten theory of curves.

It is this last theory that brought our attention to a similar type of integrals. We study moduli spaces of admissible covers, a natural compactification of the Hurwitz scheme. 
It has been shown by Abramovich, Corti and Vistoli [1] that these spaces are smooth Deligne-Mumford stacks. A class of natural intersection numbers on these spaces, parallel (and we believe related) to the structure coefficients of the Topological Quantum Field Theory in Bryan-Pandharipande [3], are obtained in the following way. Consider the diagram of stacks

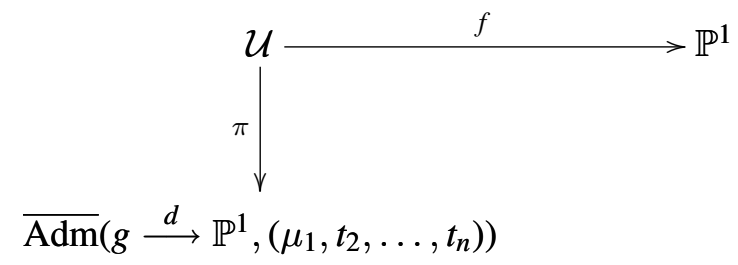

where

- $\overline{\operatorname{Adm}}\left(g \stackrel{d}{\longrightarrow} \mathbb{P}^{1},\left(\mu_{1}, t_{2}, \ldots, t_{n}\right)\right)$ denotes the space of (connected) genus $g$, degree $d$, admissible covers with ramification $\left(\mu_{1}, t_{2}, \ldots, t_{n}\right)$, which we will discuss at length in Section 1.

- we consider covers that have one arbitrary ramification point $\mu_{1}$; all other ramification is simple ( $t$ stands for transposition);

- $\mathcal{U}$ is the universal family;

- $f$ is "morally" the universal cover map (1);

Now define the class of integrals

$$
I_{d}^{\mu}(g):=\int_{\overline{\operatorname{Adm}}\left(g \stackrel{d}{\longrightarrow} \mathbb{P}^{1},\left(\mu_{1}, t_{2}, \ldots, t_{n}\right)\right)} \operatorname{ev}_{1}^{*}(\infty) \cap c_{2 g+d-1}\left(R^{1} \pi_{*} f^{*}\left(\mathcal{O}_{\mathbb{P}^{1}} \oplus \mathcal{O}_{\mathbb{P}^{1}}(-1)\right)\right),
$$

where $\mathrm{ev}_{1}$ is evaluation at the first marked point (2).

It is an elementary dimension count to show that the only non vanishing integrals must have $\mu=(d)$, that is, full ramification over the point $\infty$. For this reason we drop the superscript $\mu$.

We want to organize all of these integrals in generating function form:

$$
\mathcal{I}_{d}(x):=\sum_{g=0}^{\infty} \frac{I_{d}(g)}{2 g+d-1 !} x^{2 g+d-1} .
$$

These integrals can be approached with techniques of localization. We follow the spirit, and also the notation, of Faber and Pandharipande [6], who pioneered and developed the fundamental ideas of auxiliary localization integrals, and of using different linearizations of line bundles as means to find relations between Hodge integrals.

We make the following conjecture. 
Conjecture For all $d \geq 1$

$$
\mathcal{I}_{d}(x)=(-1)^{d-1} \frac{1}{d} \frac{\left(2 \sin \left(\frac{x}{2}\right)\right)^{d}}{2 \sin \left(\frac{d x}{2}\right)} .
$$

The conjecture is trivially true for $d=1$. In this paper we prove it for $d=2,3$. Different strategies are required to prove these two results. In degree 2 we exploit the fact that generic ramification is, in fact, full ramification. In degree 3 we prove the result by means of an auxiliary integral that we know to vanish; we can obtain the auxiliary integral precisely because full ramification can be thought of as a degeneration of simple ramification.

The strategy adopted in degree 3 should in principle work in higher degrees as well. The problem in a direct computation is that the combinatorial complexity, which is modest in the two cases we examine, grows dramatically fast.

As a corollary of these computations we obtain generating functions for another interesting class of integrals:

$$
J_{d}(g):=\int_{\overline{\operatorname{Adm}}\left(g \stackrel{d}{\longrightarrow} \mathbb{P}^{1},\left(t_{1}, t_{2}, \ldots, t_{2 g+2 d-2}\right)\right)} c_{2 g+2 d-2}\left(R^{1} \pi_{*} f^{*}\left(\mathcal{O}_{\mathbb{P}^{1}}(-1) \oplus \mathcal{O}_{\mathbb{P}^{1}}(-1)\right)\right) .
$$

In genus 0, we recover the Aspinwall-Morrison formula.

\section{Acknowledgements}

I am grateful first and foremost to my advisor, Aaron Bertram, for his constant support, motivation, and expert guidance. I also thank Y P Lee and Ravi Vakil for carefully listening to my arguments and providing useful feedback.

\section{Admissible covers}

Moduli spaces of admissible covers are a "natural" compactification of the Hurwitz scheme. The fundamental idea is that, in order to understand limit covers, we allow the base curve to degenerate together with the cover. Branch points are not allowed to "come together"; as two or more branch points tend to collide, a new component of the base curve sprouts from the point of collision, and the points transfer onto it. Similarly, upstairs the cover splits into a nodal cover.

Now more formally: let $\left(X, p_{1}, \ldots, p_{r}\right)$ be an $r$-pointed nodal curve of genus $g$. 
Definition 1 An admissible cover $\pi: E \longrightarrow X$ of degree $d$ is a finite morphism satisfying the following:

(1) $E$ is a connected nodal curve.

(2) Every node of $E$ maps to a node of X.

(3) The restriction of $\pi: E \longrightarrow X$ to $X \backslash\left(p_{1}, \ldots, p_{r}\right)$ is étale of constant degree $d$.

(4) Over a node, locally in analytic coordinates, $X, E$ and $\pi$ are described as follows:

$$
\begin{aligned}
& E: e_{1} e_{2}=a, \\
& X: x_{1} x_{2}=a^{n}, \\
& \pi: x_{1}=e_{1}^{n}, \quad x_{2}=e_{2}^{n} .
\end{aligned}
$$

Moduli spaces of admissible covers were introduced originally by Harris and Mumford in [9]. Intersection theory on these spaces was for a long time extremely hard and mysterious, mostly because they are in general not normal, even if the normalization is always smooth. Only recently in [1], Abramovich, Corti and Vistoli exhibit this normalization as the stack of balanced stable maps of degree 0 from twisted curves to the classifying stack $\mathcal{B} S_{d}$. This way they attain both the smoothness of the stack and a nice moduli-theoretic interpretation of it.

We will abuse notation and refer to the Abramovich-Corti-Vistoli spaces as admissible covers. We will be interested in admissible covers of $\mathbb{P}^{1}$. In order to estabilish notation, let us recall our basic definitions:

Definition 2 Fix $d \geq 1$, and let $\mu_{1}, \ldots, \mu_{n}$ be partitions of $d$. We denote by

$$
\overline{\operatorname{Adm}}\left(g \stackrel{d}{\longrightarrow} 0,\left(\mu_{1}, \ldots, \mu_{n}\right)\right)
$$

the connected component of the stack of balanced stable maps of degree 0 from a genus $0, n$-pointed twisted curve to $\mathcal{B} S_{d}$ characterized by the following conditions:

(1) the associated admissible cover (according to the construction in [1, page 3566]) is a nodal curve of genus $g$.

(2) let $x_{1}, \ldots, x_{n}$ be the marks on the base curve; the ramification profile over $x_{i}$ is required to be of type $\mu_{i}$.

We call this the stack of admissible covers of degree $d$ and genus $g$ of a genus 0 curve. 
This is either empty or a smooth stack of dimension $n-3=2 g+2 d+n+\sum \ell\left(\mu_{i}\right)-n d-5$, where $\ell\left(\mu_{i}\right)$ denotes the length of the partition $\mu_{i}$. It admits two natural maps into moduli spaces of curves, as represented in the following diagram:

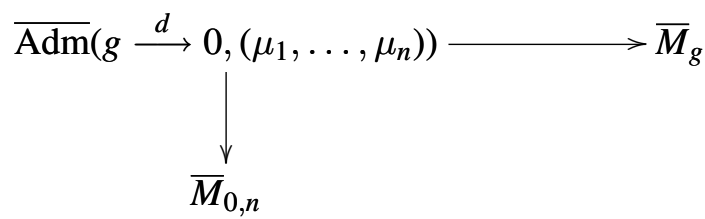

In particular, the vertical map has finite fibers.

We also are interested in fixing a parametrization of the base $\mathbb{P}^{1}$. The objects we parametrize are the same as above, but the equivalence relation is stricter: we consider two covers $E_{1} \rightarrow \mathbb{P}^{1}, E_{2} \rightarrow \mathbb{P}^{1}$ equivalent if there is an isomorphism $\varphi: E_{1} \rightarrow E_{2}$ that makes the natural triangle commute. In other words, we are not allowed to act on the base with an automorphism of $\mathbb{P}^{1}$.

Definition 3 We denote by

$$
\overline{\operatorname{Adm}}\left(g \stackrel{d}{\longrightarrow} \mathbb{P}^{1},\left(\mu_{1}, \ldots, \mu_{n}\right)\right)
$$

the stack of admissible covers of degree $d$ of (a parametrized) $\mathbb{P}^{1}$ by curves of genus $g$, with $\mathrm{n}$ specified branch points having ramification profile $\mu_{1}, \ldots, \mu_{n}$.

We construct the space of parametrized admissible covers as the stack of balanced stable maps of degree $d$ ! from the category of genus $0, n$-pointed twisted curves to the stack quotient $\left[\mathbb{P}^{1} / S_{d}\right]$, where $S_{d}$ acts trivially on $\mathbb{P}^{1}$. This is but a slight variation to the Abramovich-Corti-Vistoli construction. Let us illustrate what happens over a geometric point $\operatorname{Spec}(\mathbb{C})$ :

A map of degree $d$ ! from the twisted curve produces a map of degree 1 from the coarse curve (and this is our desired parametrization of one special genus 0 twig on the base), a principal $S_{d}$ bundle over the twisted curve and an $S_{d}$ equivariant map to $\mathbb{P}^{1}$ (this data characterizes the admissible cover). Two admissible covers are equivalent if there is an automorphism of the twisted curve that makes them commmute. In doing so, the degree 1 map to $\mathbb{P}^{1}$ has to be respected, so only the non-parametrized twigs are free to be acted upon by automorphisms. This is either empty or a smooth stack of dimension 


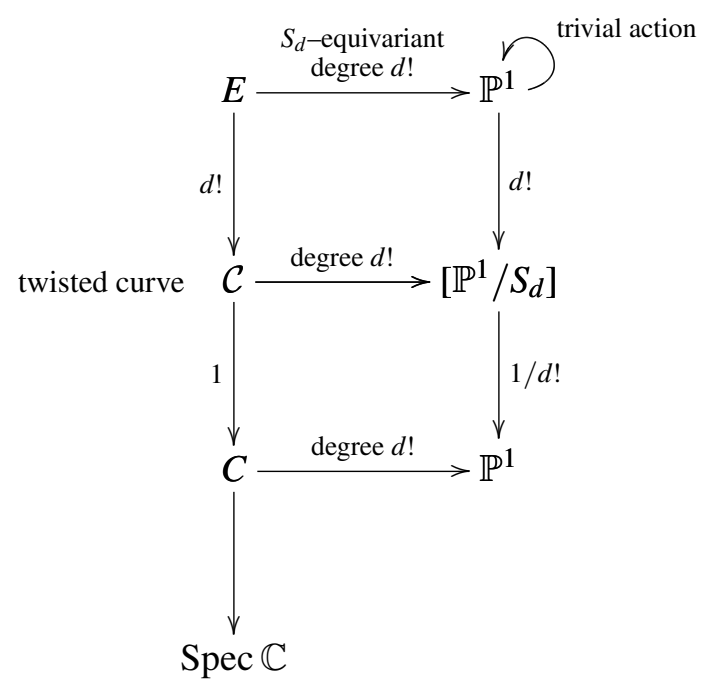

Figure 1: The stack of admissible covers of a parametrized $\mathbb{P}^{1}$

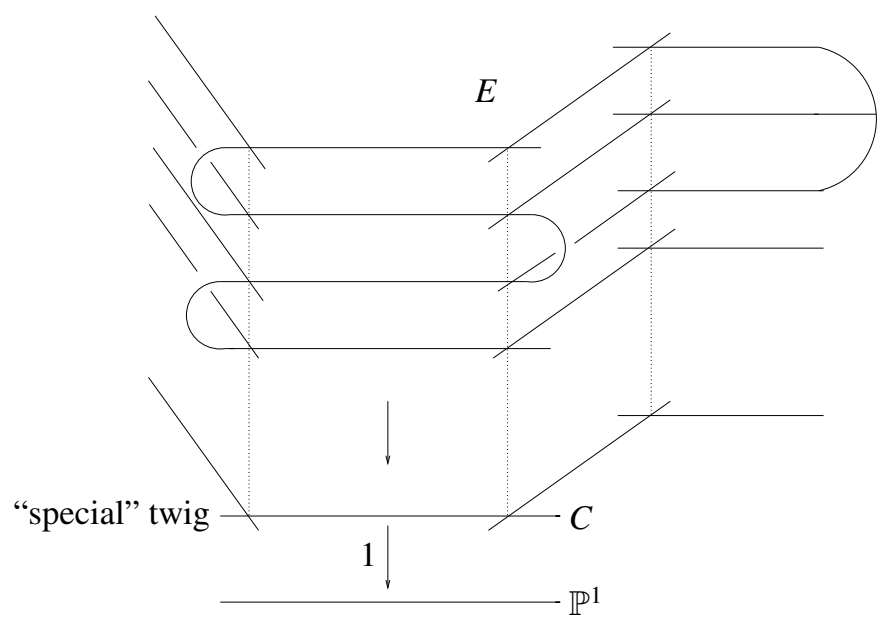

Figure 2: Schematic depiction of an admissible cover of a parametrized $\mathbb{P}^{1}$

$n=2 g+2 d+n+\sum \ell\left(\mu_{i}\right)-n d-2$, admitting two natural morphisms

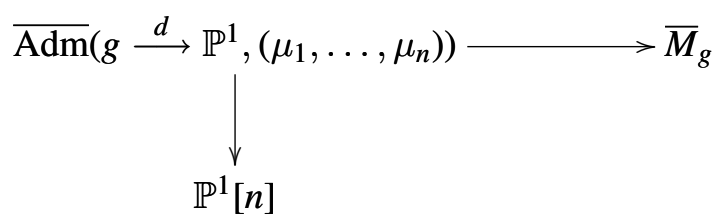


The map to $\bar{M}_{g}$ just looks at the source curve forgetting the cover map. The vertical morphism, taking values in the Fulton-MacPherson configuration space of $n$ points in $\mathbb{P}^{1}$, looks instead at the target curve, and at the (ordered) branch points.

The stacks of admissible covers of $\mathbb{P}^{1}$ admit a universal family $\mathcal{U}$, and a universal cover map $\mu$. The cover map takes values in a stack $\mathcal{X}$, that is a family over the moduli space. The fiber over a moduli point consists of a nodal, genus 0 curve, with one special irreducible component. The universal cover map can be followed by a map $\varepsilon$, that contracts all secondary twigs and takes values in $\overline{\operatorname{Adm}} \times \mathbb{P}^{1}$. Finally the right projection lands us in $\mathbb{P}^{1}$.

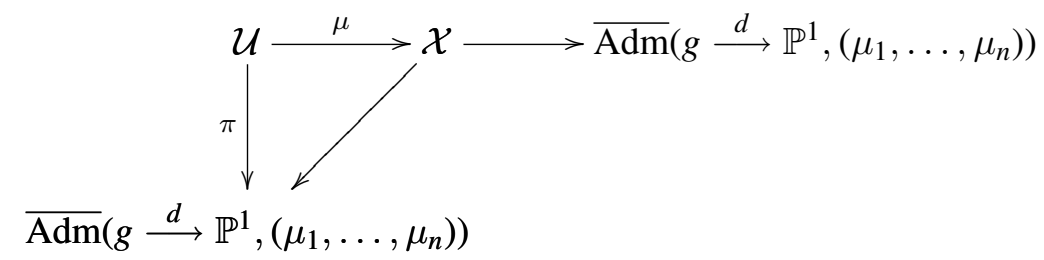

We call $f$ the composition of the three horizontal maps.

The universal family can be itself interpreted as a moduli space of admissible covers. If we think of admissible covers as of stable maps from a twisted curve, then we obtain a Universal family by adding a mark to the twisted curve and requiring trivial ramification over it. Let us denote with (1) the partition $(1, \ldots, 1)$ of $d$, representing an unramified point. Then,

$$
\mathcal{U}=\overline{\operatorname{Adm}}\left(g \stackrel{d}{\longrightarrow} \mathbb{P}^{1},\left(\mu_{1}, \ldots, \mu_{n},(1)\right)\right) .
$$

We can define $n$ tautological sections

$$
\sigma_{i}: \overline{\operatorname{Adm}}\left(g \stackrel{d}{\longrightarrow} \mathbb{P}^{1},\left(\mu_{1}, \ldots, \mu_{n}\right)\right) \longrightarrow \overline{\operatorname{Adm}}\left(g \stackrel{d}{\longrightarrow} \mathbb{P}^{1},\left(\mu_{1}, \ldots, \mu_{n},(1)\right)\right)
$$

of the natural forgetful map. The image of the $i$ th section consists of covers where a new rational component has sprouted from the $i$ th marked point. The marked points (1) and $\mu_{i}$ have transferred onto this twig. Over this twig we find $\ell\left(\mu_{i}\right)$ copies of $\mathbb{P}^{1}$ fully ramified over the attaching point and over the marked point $\mu_{i}$.

Finally we can define the natural evaluation maps

$$
\mathrm{ev}_{i}:=f \circ \sigma_{i}: \overline{\operatorname{Adm}}\left(g \stackrel{d}{\longrightarrow} \mathbb{P}^{1},\left(\mu_{1}, \ldots, \mu_{n}\right)\right) \longrightarrow \mathbb{P}^{1} .
$$



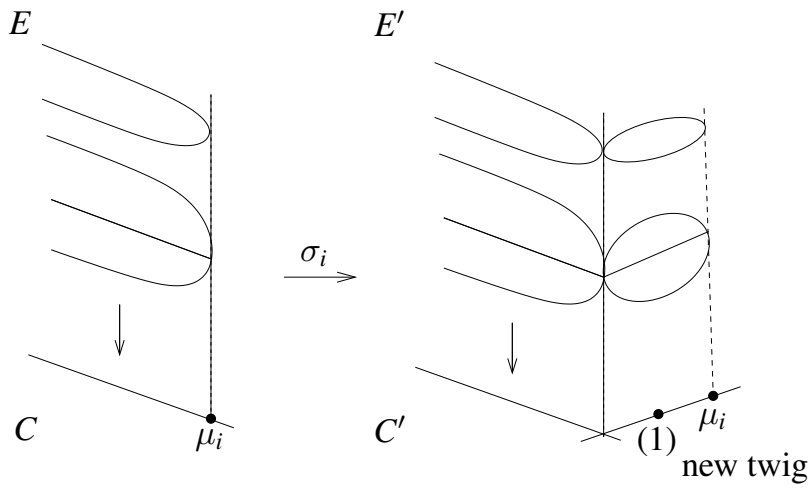

Figure 3: The tautological section $\sigma_{i}$

\subsection{The boundary}

The boundary of spaces of admissible covers can be described in terms of admissible cover spaces of possibly lower degree or genus. In the case of admissible covers of a parametrized $\mathbb{P}^{1}$, the boundary will involve also admissible covers of an unparametrized genus 0 curve. In Figure 1, for example, we can obtain the depicted admissible cover by "gluing together" one admissible cover of a parametrized $\mathbb{P}^{1}$ (the cover of the special twig) and three admissible covers of an irreducible genus 0 curve. It would be very tempting to conclude that the irreducible boundary components of an admissible cover space are actually products of other admissible cover spaces; however we need to be very careful, and consider the contribution to the stack structure given by automorphisms.

To illustrate this point let us carefully analyze the gluing map. For simplicity of exposition, let's glue at a fully ramified point:

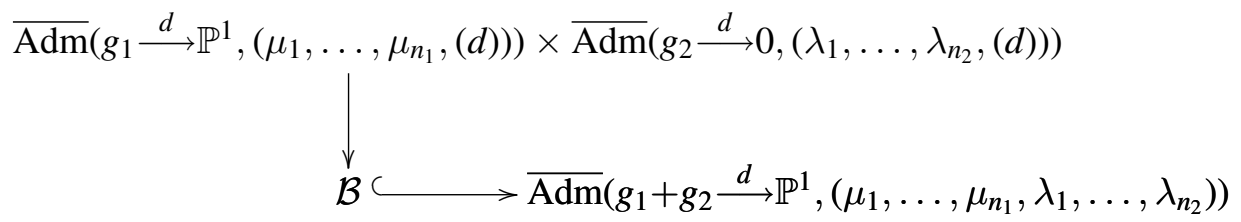

We claim that the vertical map is an étale map of stacks of degree $1 / d$. Let us look at a point $[E \rightarrow X]$ of $\mathcal{B}$ : we observe that it admits a unique preimage $\left(\left[E_{1} \rightarrow \mathbb{P}^{1}\right],\left[E_{2} \rightarrow\right.\right.$ $\left.X_{2}\right]$ ), and we count the automorphisms of the preimage modulo automorphisms pulledback from below. In local analytic coordinates around the node, the cover is described 
as

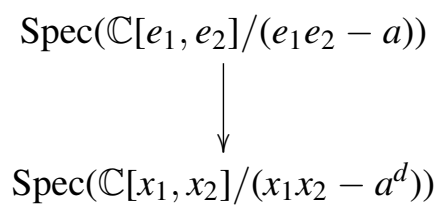

by the local equations $x_{1}=e_{1}^{d}, x_{2}=e_{2}^{d}$. Modding out by automorphisms of the "glued" cover is equivalent to requiring the first coordinate $e_{1}$ to remain untouched. It is then evident that what we have left are $d$ distinct automorphisms, consisting in multiplying $e_{2}$ by a $d^{\text {th }}$ root of unity. This establishes our claim.

Now if we want to glue two branch points with ramification profile $\eta=\left(d_{1}, \ldots, d_{k_{\mu}}\right)$, with all the $d_{i}$ 's distinct, the situation will be analogous. The gluing map

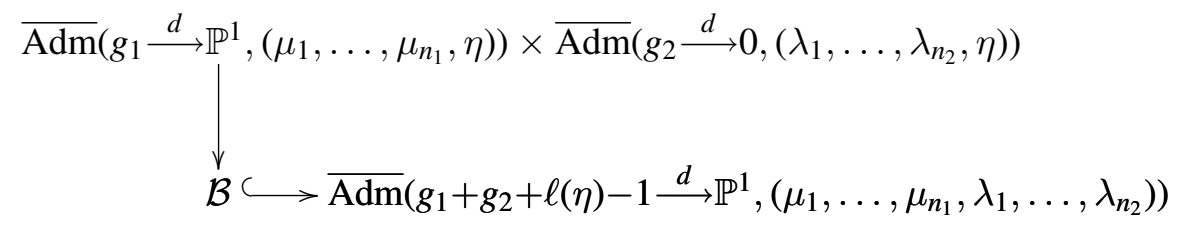

is an étale map of stacks of degree $1 /\left(d_{1} \cdot \ldots \cdot d_{k_{\mu}}\right)$.

For the purposes of this paper, this is all we are concerned with. For the sake of a more complete exposition, we briefly describe what the situation for a general partition $\eta$ is. Let

$$
\eta=\left(\left(\eta^{1}\right)^{m_{1}}, \ldots,\left(\eta^{k}\right)^{m_{k}}\right) .
$$

The gluing map (3) is an étale map of stacks of degree

$$
\frac{1}{\prod\left(\eta^{i}\right)^{m_{i}} m_{i} !} \text {. }
$$

There is a little bit of combinatorial subtlety to be dealt with to obtain this last result. In order to be able to even define a gluing map we must introduce markings on the covers. Ionel develops the theory of these spaces in [10]; our spaces are étale quotients of Ionel spaces. The gluing map is well defined on the level of Ionel spaces, and it descends to (4).

\subsection{Tautological classes}

We are interested in describing some "tautological" intersection classes on the stack of admissible covers of an unparametrized genus 0 curve: in particular we want to endow 
our space with analogues of $\lambda$ and $\psi$ classes. To do so, we will simply pull-back these classes from the appropriate moduli spaces.

Recall the forgetful map

$$
\overline{\operatorname{Adm}}\left(g \stackrel{d}{\longrightarrow} 0,\left(\mu_{1}, \ldots, \mu_{n}\right)\right) \stackrel{s}{\longrightarrow} \bar{M}_{g} .
$$

The tautological class $\lambda_{i} \in A^{i}\left(\bar{M}_{g}\right)$ is defined to be the $i$ th Chern class of the Hodge bundle $\mathbb{E}$.

Definition 4 The tautological class $\lambda_{i}^{\operatorname{Adm}} \in A^{i}\left(\overline{\operatorname{Adm}}\left(g \stackrel{d}{\longrightarrow} 0,\left(\mu_{1}, \ldots, \mu_{n}\right)\right)\right)$ is defined to be the $i$ th Chern class of the pull-back of the Hodge bundle via the map $s$ :

$$
\lambda_{i}^{\mathrm{Adm}}:=s^{*}\left(\lambda_{i}\right)
$$

We will drop the superscript "Adm" and simply write $\lambda_{i}$ whenever there is no risk of confusion.

Let us now look at another natural map

$$
\overline{\operatorname{Adm}}\left(g \stackrel{d}{\longrightarrow} 0,\left(\mu_{1}, \ldots, \mu_{n}\right)\right) \stackrel{t}{\longrightarrow} \overline{\mathcal{M}}_{0, n} .
$$

The stack $\overline{\mathcal{M}}_{0, n}$ is the moduli space of twisted $n$-pointed curves of genus 0 .

Let $\overline{\mathcal{M}}_{0, n+1} \stackrel{\pi}{\longrightarrow} \overline{\mathcal{M}}_{0, n}$ be the universal family over this stack, $\omega_{\pi} \longrightarrow \overline{\mathcal{M}}_{0, n+1}$ be the relative dualizing sheaf and $\sigma_{i}$ the $i$ th tautological section. Then $\psi_{i} \in A^{1}\left(\overline{\mathcal{M}}_{0, n}\right)$ is defined to be the first Chern class of $\sigma_{i}^{*}\left(\omega_{\pi}\right)$.

Definition 5 The tautological class $\psi_{i}^{\operatorname{Adm}} \in A^{1}\left(\overline{\operatorname{Adm}}\left(g \stackrel{d}{\longrightarrow} 0,\left(\mu_{1}, \ldots, \mu_{n}\right)\right)\right)$ is defined to be the pull-back of the analogous class via the map $t$ :

$$
\psi_{i}^{\mathrm{Adm}}:=t^{*}\left(\psi_{i}\right)
$$

Again, the superscript will be dropped unless needed for clarity. We can also view $\psi$ classes in a more intrinsic fashion. Consider

- the space

$$
\overline{\operatorname{Adm}}\left(g \stackrel{d}{\longrightarrow} 0,\left(\mu_{1}, \ldots, \mu_{n},(1)\right)\right)
$$

where we have added a trivial ramification condition;

- the forgetful map

$$
\pi_{(1)}: \overline{\operatorname{Adm}}\left(g \stackrel{d}{\longrightarrow} 0,\left(\mu_{1}, \ldots, \mu_{n},(1)\right)\right) \longrightarrow \overline{\operatorname{Adm}}\left(g \stackrel{d}{\longrightarrow} 0,\left(\mu_{1}, \ldots, \mu_{n}\right)\right) ;
$$


- the $i$ th tautological section

$$
\sigma_{i}: \overline{\operatorname{Adm}}\left(g \stackrel{d}{\longrightarrow} 0,\left(\mu_{1}, \ldots, \mu_{n}\right)\right) \longrightarrow \overline{\operatorname{Adm}}\left(g \stackrel{d}{\longrightarrow} 0,\left(\mu_{1}, \ldots, \mu_{n},(1)\right)\right) .
$$

Lemma 6 The class $-\psi_{i} \in A^{1}\left(\overline{\operatorname{Adm}}\left(g \stackrel{d}{\longrightarrow} 0,\left(\mu_{1}, \ldots, \mu_{n}\right)\right)\right)$ is the first Chern class of the normal bundle to the image of the section $\sigma_{i}$.

Proof Observe the following commutative diagram:

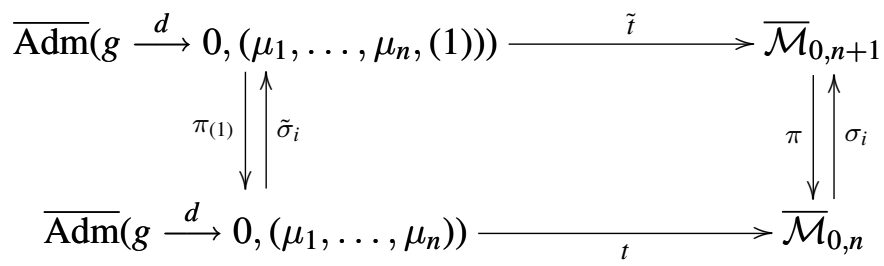

We know from Abramovich, Corti and Vistoli [1, page 3561] that the maps $t$ and $\tilde{t}$ are étale onto their image. Further, the diagram is cartesian. Now our lemma follows from the analogous statement on $\overline{\mathcal{M}}_{0, n}$ :

$$
-\psi_{i}^{\mathrm{Adm}}=t^{*}\left(-\psi_{i}\right)=c_{1}\left(t^{*} \sigma_{i}^{*} N_{\sigma_{i}}\right)=c_{1}\left(\tilde{\sigma}_{i}^{*} \tilde{t}^{*} N_{\sigma_{i}}\right)=c_{1}\left(\tilde{\sigma}_{i}^{*} N_{\tilde{\sigma}_{i}}\right)
$$

\section{Localization}

The main tool for evaluating our integrals is the Atiyah-Bott localization theorem [2]. Consider the 1 dimensional algebraic torus $\mathbb{C}^{*}$, and recall that the $\mathbb{C}^{*}$-equivariant Chow ring of a point is a polynomial ring in one variable:

$$
A_{\mathbb{C}^{*}}^{*}(\{p t\}, \mathbb{C})=\mathbb{C}[\hbar]
$$

Let $\mathbb{C}^{*}$ act on a smooth, proper stack $X$, denote by $i_{k}: F_{k} \hookrightarrow X$ the irreducible components of the fixed locus for this action and by $N_{F_{k}}$ their normal bundles. The natural map:

$$
\begin{aligned}
A_{\mathbb{C}^{*}}^{*}(X) \otimes \mathbb{C}(\hbar) & \longrightarrow \sum_{k} A_{\mathbb{C}^{*}}^{*}\left(F_{k}\right) \otimes \mathbb{C}(\hbar) \\
\alpha & \longmapsto \frac{i_{k}^{*} \alpha}{c_{t o p}\left(N_{F_{k}}\right)} .
\end{aligned}
$$

is an isomorphism. Pushing forward equivariantly to the class of a point, we obtain the Atiyah-Bott integration formula

$$
\int_{[X]} \alpha=\sum_{k} \int_{\left[F_{k}\right]} \frac{i_{k}^{*} \alpha}{c_{t o p}\left(N_{F_{k}}\right)} .
$$




\subsection{Our set-up}

Let $\mathbb{C}^{*}$ act on a 2-dimensional vector space $V$ via

$$
t \cdot\left(z_{0}, z_{1}\right)=\left(t z_{0}, z_{1}\right)
$$

This action descends on $\mathbb{P}^{1}$, with fixed points $0=(1: 0)$ and $\infty=(0: 1)$. An equivariant lifting of $\mathbb{C}^{*}$ to a line bundle $L$ over $\mathbb{P}^{1}$ is uniquely determined by its weights $\left\{L_{0}, L_{\infty}\right\}$ over the fixed points.

The canonical lifting of $\mathbb{C}^{*}$ to the tangent bundle of $\mathbb{P}^{1}$ has weights $\{1,-1\}$.

The action on $\mathbb{P}^{1}$ induces an action on the moduli spaces of admissible covers to a parametrized $\mathbb{P}^{1}$ simply by postcomposing the cover map with the automorphism of $\mathbb{P}^{1}$ defined by $t$.

The fixed loci for the induced action on the moduli space consist of admissible covers such that anything "interesting" (ramification, nodes) happens over 0 and $\infty$, or on "non-special" twigs that attach to the main $\mathbb{P}^{1}$ at 0 or $\infty$.

\subsection{Restricting Chow classes to the fixed loci}

We want to compute the restriction to various fixed loci of the top Chern class of the bundle

$$
E=R^{1} \pi_{*} f^{*}\left(\mathcal{O}_{\mathbb{P}^{1}} \oplus \mathcal{O}_{\mathbb{P}^{1}}(-1)\right) .
$$

The top Chern class $c_{2 g+d-1}(E)$ splits as

$$
c_{2 g+d-1}(E)=c_{g}\left(R^{1} \pi_{*} f^{*} \mathcal{O}_{\mathbb{P}^{1}}\right) c_{g+d-1}\left(R^{1} \pi_{*} f^{*} \mathcal{O}_{\mathbb{P}^{1}}(-1)\right),
$$

so we will analyze the two terms separately.

There is a standard technique to carry out these computations. To avoid an overwhelmingly cumbersome notation, we choose to show it only in a particular example, that will be the most important for our purposes.

Let's consider the fixed locus $F_{g_{1} g_{2}}$, consisting of covers where the main $\mathbb{P}^{1}$ is ramified over 0 and $\infty$ and curves of genus $g_{1}$ and $g_{2}$ are attached on either side. A point in this fixed locus is represented in Figure 4, where we denote by $X$ the nodal curve, $C_{1}$ and $C_{2}$ the irreducible components over 0 and $\infty$. 


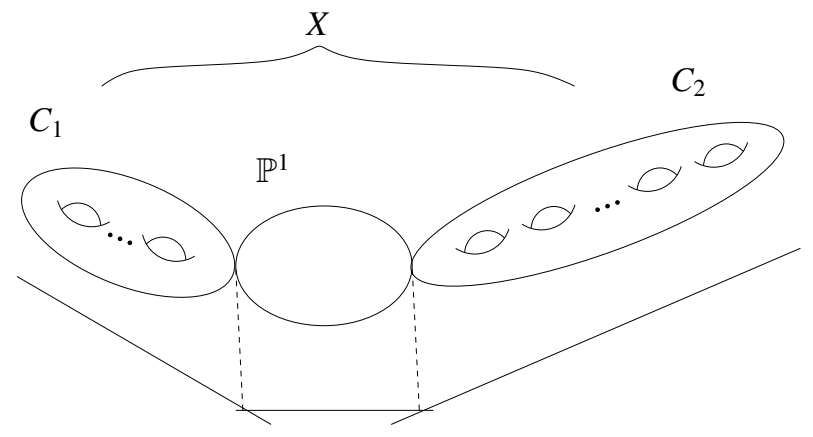

Figure 4: The fixed locus $F_{g_{1}, g_{2}}$

The starting point in analyzing the restriction of the bundle $E$ to this fixed locus is the classical normalization sequence:

$$
0 \longrightarrow \mathcal{O}_{X} \longrightarrow \mathcal{O}_{C_{1}} \oplus \mathcal{O}_{\mathbb{P}^{1}} \oplus \mathcal{O}_{C_{2}} \longrightarrow \mathbb{C}_{n_{1}} \oplus \mathbb{C}_{n_{2}} \longrightarrow 0 .
$$

Term $1\left(c_{g}\left(R^{1} \pi_{*} f^{*} \mathcal{O}_{\mathbb{P}^{1}}\right)\right)$ It suffices to analyze the long exact sequence in cohomology associated to the normalization sequence

$$
\begin{aligned}
0 \longrightarrow h^{0}\left(\mathcal{O}_{X}\right) \longrightarrow h^{0}\left(\mathcal{O}_{C_{1}}\right) \oplus h^{0}\left(\mathcal{O}_{\mathbb{P}^{1}}\right) \oplus h^{0}\left(\mathcal{O}_{C_{2}}\right) & \longrightarrow \mathbb{C}_{n_{1}} \oplus \mathbb{C}_{n_{2}} \\
& \longrightarrow h^{1}\left(\mathcal{O}_{X}\right) \longrightarrow h^{1}\left(\mathcal{O}_{C_{1}}\right) \oplus h^{1}\left(\mathcal{O}_{C_{2}}\right) \longrightarrow 0
\end{aligned}
$$

Assume that $\mathcal{O}_{\mathbb{P}^{1}}$ is linearized with weights $\{\alpha, \alpha\}$. Then

$$
c_{g}\left(R^{1} \pi_{*} f^{*} \mathcal{O}_{\mathbb{P}^{1}}\right)=(-)^{g} \Lambda_{g_{1}}(-\alpha) \Lambda_{g_{2}}(-\alpha),
$$

where the following notational convention holds:

$$
\Lambda_{g}(n)=\sum(n \hbar)^{i} \lambda_{g-i}
$$

The reason for switching from $\alpha$ to $-\alpha$ is that $h^{1}(\mathcal{O})$ are the fibers of the dual bundle to the Hodge bundle, hence the odd degree Chern classes will have a negative sign.

Term $2\left(c_{g+d-1}\left(R^{1} \pi_{*} f^{*} \mathcal{O}_{\mathbb{P}^{1}}(-1)\right)\right)$ In this case we first want to tensor the normalization sequence by $f^{*} \mathcal{O}_{\mathbb{P}^{1}}(-1)$, and then proceed to analyze the long exact sequence in cohomology:

$$
\begin{aligned}
0 \longrightarrow h^{0}\left(\mathcal{O}_{C_{1}}\right) \oplus h^{0}\left(\mathcal{O}_{C_{2}}\right) \longrightarrow \mathbb{C}_{n_{1}} \oplus \mathbb{C}_{n_{2}} \\
\longrightarrow h^{1}\left(f^{*} \mathcal{O}_{\mathbb{P}^{1}}(-1)\right) \longrightarrow h^{1}\left(\mathcal{O}_{C_{1}}\right) \oplus h^{1}\left(\mathcal{O}_{\mathbb{P}^{1}}(-d)\right) \oplus h^{1}\left(\mathcal{O}_{C_{2}}\right) \longrightarrow 0
\end{aligned}
$$


Now, having linearized $\mathcal{O}_{\mathbb{P}^{1}}(-1)$ with weights $\{\beta, \beta+1\}$,

$$
c_{g+d-1}\left(R^{1} \pi_{*} f^{*} \mathcal{O}_{\mathbb{P}^{1}}(-1)\right)=(-)^{g} \Lambda_{g_{1}}(-\beta) \Lambda_{g_{2}}(-\beta-1) \hbar^{d-1} \prod_{1}^{d-1}\left(\beta+\frac{i}{d}\right) .
$$

The last term in our contribution, coming from $h^{1}\left(\mathcal{O}_{\mathbb{P}^{1}}(-d)\right)$, is explained in the following way. Consider a degree $d$ map from $\mathbb{P}^{1}$ to $\mathbb{P}^{1}$. The target curve is given the natural $\mathbb{C}^{*}$ action, and the tautological bundle is linearized with weights $\{\beta, \beta+1\}$. Now let $x$ and $z$ be local coordinates around 0 for, respectively, the target and the source curve. The expression of the map in local coordinates is

$$
x=z^{d} .
$$

We see then that $z$ must have weight $-1 / d$. The vector space $h^{1}\left(\mathcal{O}_{\mathbb{P}^{1}}(-d)\right)$ is $(d-1)$ dimensional and generated, in local coordinates, by the sections $\left\{1 / z, 1 / z^{2}, \ldots, 1 / z^{d-1}\right\}$. The line bundle over moduli with these fibers is trivial, because $\mathbb{P}^{1}$ is rigid, but it is linearized with weights $\beta+i / d ; \beta$ coming from the weight of the trivialization of the pullback of $\mathcal{O}_{\mathbb{P}^{1}}(-1)$ in the chart over $0, i / d$ from the section $1 / z^{i}$. Notice that if you were to reproduce this computation using a local cohordinate over $\infty$ instead, the corresponding weights would now be $(\beta+1)-(d-i) / d$, which are exactly the same.

\subsection{The Euler class of the normal bundle to the fixed loci}

The standard way to carry out this computation is to analyze the deformation long exact sequence, and identify the fiber of the normal bundle to a fixed locus at a particular moduli point to the moving part (the part where the $\mathbb{C}^{*}$ action doesn't lift trivially) of the tangent space to the moduli space (corresponding to the space of first order deformations of the admissible cover in question). It's shown by Abramovich, Corti and Vistoli [1, page 3561] that the deformation theory of admissible covers corresponds exactly to the deformation theory of the base, genus 0 , twisted curve. The reason for this is that admissible covers are étale covers (in fact principal $S_{d}$-bundles) of the base twisted curve.

Deformations of a genus 0 nodal twisted curve are described as follows: first of all, we can deal with one node at a time. For one given node, there are two different potential contributions:

- the contribution from moving the node on the main $\mathbb{P}^{1}$. Doing this infinitesimally means moving along the tangent space to the attaching point on the main $\mathbb{P}^{1}$. 
Again, the bundle with fiber the tangent space over a given point of $\mathbb{P}^{1}$ is a trivial bundle, but in equivariant cohomology it can have a purely equivariant first Chern class, according to the linearization of the fibers. In our particular case, the tangent bundle has weight 1 over 0 and -1 over $\infty$, thus producing a contribution of $\hbar$ for moving a node around 0 , of $-\hbar$ for moving a node around $\infty$

- the contribution from smoothing the node. It corresponds to the first Chern class of the tensor product of the tangent spaces at the attaching points of the two curves. Again, we get a $\pm \hbar$ contribution from the point on the main $\mathbb{P}^{1}$; the other attaching point $x$, on the other hand, contributes, by definition, a $-\psi_{x}$ class.

\section{Degree 2}

We now carry out the explicit computation of the integral

$$
I_{2}(g)=\int_{\overline{\operatorname{Adm}}\left(g \stackrel{2}{\longrightarrow} \mathbb{P}_{\mathbb{C}}^{1},\left(t_{1}, t_{2}, \ldots, t_{2 g+2}\right)\right)} \operatorname{ev}_{1}^{*}(\infty) \cap c_{2 g+1}\left(R^{1} \pi_{*} f^{*}\left(\mathcal{O}_{\mathbb{P}^{1}} \oplus \mathcal{O}_{\mathbb{P}^{1}}(-1)\right)\right),
$$

for all genera, and express the result in generating function form:

$$
\mathcal{I}_{2}(x)=\sum_{g=0}^{\infty}\left(\frac{I_{2}(g)}{2 g+1 !}\right) x^{2 g+1} .
$$

\subsection{The strategy}

It is important to notice that, while the final result is independent of the choice of the lifting of the $\mathbb{C}^{*}$ action to the vector bundle $E=R^{1} \pi_{*} f^{*}\left(\mathcal{O}_{\mathbb{P}^{1}} \oplus \mathcal{O}_{\mathbb{P}^{1}}(-1)\right)$, the intermediate calculations are not. This is in fact the heart of our strategy. We choose two different specific linearizations with the twofold objective of

- limiting a priori the number and the combinatorial complexity of the contributing fixed loci;

- obtaining, by equating the calculations with the two linearizations, a recursive formula for genus $g$ integrals in term of lower genus data. 


\subsection{The localization set-up}

We induce different linearizations on the bundle $E$ by choosing different liftings of the $\mathbb{C}^{*}$ action on the bundles $\mathcal{O}_{\mathbb{P}^{1}}$ and $\mathcal{O}_{\mathbb{P}^{1}}(-1)$. Recall that a linearization of a line bundle over $\mathbb{P}^{1}$ is determined by the weights of the fixed fibers representations.

Linearization A We choose to linearize the two bundles as indicated in the following table:

\begin{tabular}{|l||c|c|}
\hline weight & over 0 & over $\infty$ \\
\hline \hline $\mathcal{O}_{\mathbb{P}^{1}}(-1)$ & -1 & 0 \\
\hline $\mathcal{O}_{\mathbb{P}^{1}}$ & 0 & 0 \\
\hline
\end{tabular}

There is only one fixed locus $F_{g}$, contributing to the localization integral, consisting in a cover of $\mathbb{P}^{1}$ fully ramified over 0 and $\infty$, and a genus $g$ curve mapping with degree 2 to an unparametrized $\mathbb{P}^{1}$ sprouting from the point 0 . Figure 5 illustrates the fixed locus, and the conventional graph notation to indicate it.
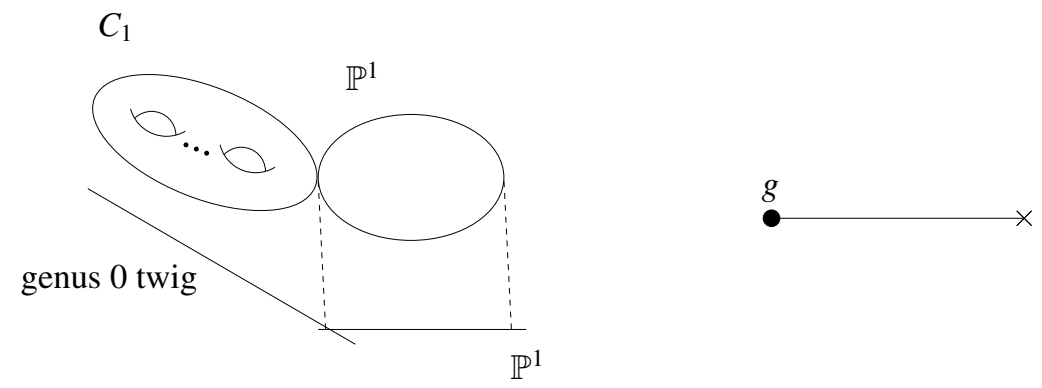

Figure 5: The fixed locus $F_{g}$.

The reason for this dramatic collapsing of the contributing fixed loci lies in some standard localization facts:

- the ramification condition required over $\infty$ implies that there can be only one connected component in the preimage of $\infty$. This translates to the fact that the localization graph can have at most 1 vertex over $\infty$;

- the weight 0 linearization of $\mathcal{O}_{\mathbb{P}^{1}}(-1)$ over $\infty$ implies that the localization graph must have valence 1 over $\infty$.

- finally, let's observe that both bundles have weight 0 over $\infty$; the restriction of our bundle to fixed loci that have contracted components over $\infty$ involves the class $\lambda_{g_{\infty}}^{2}$, that vanishes for $g>0$ by a famous result by Mumford [11]. The 
only option is then to have genus 0 over infinity. But a genus zero curve with only two special points is instable, and hence must be contracted.

Linearization B We choose to linearize the bundles with weights:

\begin{tabular}{|l||c|c|}
\hline weight & over 0 & over $\infty$ \\
\hline \hline $\mathcal{O}_{\mathbb{P}^{1}}(-1)$ & -1 & 0 \\
\hline $\mathcal{O}_{\mathbb{P}^{1}}$ & 1 & 1 \\
\hline
\end{tabular}

In this case the analysis of the possibly contributing fixed loci is similar, except we can't appeal to Mumford's relation any more. Hence our fixed loci will consist of a copy of $\mathbb{P}^{1}$ ramified over 0 and $\infty$, with two curves of genus $g_{1}, g_{2}$ attached on either side. (And, of course, $g_{1}+g_{2}=g$ ). These are the loci $F_{g_{1}, g_{2}}$ described in Figure 4.

\subsection{Explicit evaluation of the integral and recursion}

Linearization A Let us first of all observe that $F_{g}$, is naturally isomorphic to $\overline{\operatorname{Adm}}\left(g \stackrel{2}{\longrightarrow} 0,\left(t_{1}, t_{2}, \ldots, t_{2 g+2}\right)\right)$. Using the computations in Section 2 , and the standard equivariant cohomology fact that $\mathrm{ev}_{1}^{*}(\infty)=-\hbar$, we obtain the explicit evaluation of our integral on this fixed locus:

$$
\begin{aligned}
I_{2}^{A}(g)=\int_{\overline{\operatorname{Adm}}\left(g \stackrel{2}{\longrightarrow} 0,\left(t_{1}, t_{2}, \ldots, t_{2 g+2}\right)\right)} \frac{\lambda_{g} \Lambda_{g}(1)(-\hbar / 2)}{\hbar(\hbar-\psi)} & \\
= & -\frac{1}{2} \int_{\overline{\operatorname{Adm}}\left(g \stackrel{2}{\longrightarrow} 0,\left(t_{1}, t_{2}, \ldots, t_{2 g+2}\right)\right)} \lambda_{g} \lambda_{g-1}+\lambda_{g} \lambda_{g-2} \psi+\cdots+\lambda_{g} \psi^{g-1} .
\end{aligned}
$$

Just as a convenient notation, let's denote the last integral by $L_{2}(g)$, so that

$$
I_{2}^{A}(g)=-\frac{1}{2} L_{2}(g)
$$

Linearization B In this case we have $g+1$ different types of fixed loci, corresponding to all possible ways of choosing an ordered pair of nonnegative integers adding to $g$. We will study separately three situations:

$F_{\cdot, g}$ This fixed locus is naturally isomorphic to $2 g+1$ disjoint copies of

$$
\overline{\operatorname{Adm}}\left(g \stackrel{2}{\longrightarrow} 0,\left(t_{1}, t_{2}, \ldots, t_{2 g+2}\right)\right) \text {. }
$$


The evaluation of the integral reads

$$
\begin{aligned}
& \int_{F_{\cdot, g}} \frac{\lambda_{g} \Lambda_{g}(-1)(-\hbar / 2)}{(-\hbar-\psi)}= \\
&-\frac{2 g+1}{2} \int_{\overline{\operatorname{Adm}}\left(g \stackrel{2}{\longrightarrow} 0,\left(t_{1}, t_{2}, \ldots, t_{2 g+2}\right)\right)} \lambda_{g} \lambda_{g-1}+\lambda_{g} \lambda_{g-2} \psi+\cdots+\lambda_{g} \psi^{g-1} \\
&=-\frac{2 g+1}{2} L_{2}(g) .
\end{aligned}
$$

$F_{g_{1}, g_{2}}, g_{1}, g_{2} \neq 0$ ) After keeping track of the combinatorics of the gluing and of the possible distributions of the marks, the integral evaluates

$$
\begin{aligned}
& \int_{F_{g_{1}, g_{2}}} \frac{\Lambda_{g_{1}}(-1) \Lambda_{g_{1}}(1) \lambda_{g_{2}} \Lambda_{g_{2}}(-1)(-\hbar / 2)}{\hbar(\hbar-\psi)(-\hbar-\psi)} \\
&=-\left(\begin{array}{c}
2 g+1 \\
2 g_{2}
\end{array}\right) \int_{\overline{\operatorname{Adm}}\left(g_{1} \stackrel{2}{\longrightarrow} 0\right)}(-)^{g_{1}} \psi^{2 g_{1}-1} \\
& \int_{\overline{\operatorname{Adm}}\left(g_{2} \stackrel{2}{\longrightarrow} 0\right)} \lambda_{g_{2}} \lambda_{g_{2}-1}+\lambda_{g_{2}} \lambda_{g_{2}-2} \psi+\cdots+\lambda_{g_{2}} \psi^{g_{2}-1} \\
&:=(-)^{g_{1}+1} \frac{1}{2}\left(\begin{array}{c}
2 g+1 \\
2 g_{2}
\end{array}\right) P_{2}\left(g_{1}\right) L_{2}\left(g_{2}\right) .
\end{aligned}
$$

To make the notation a little lighter we omitted the marked points (that are still there, though). Also we choose to denote with $P_{2}$ the integral of $\psi_{t}$ to the top power.

$F_{g,}$ This is the same fixed locus encountered in the computations with linearization A. However, the contribution in this case will be quite different:

$$
\begin{aligned}
& \int_{F_{g, \cdot}} \frac{\Lambda_{g}(1) \Lambda_{g}(-1)(-\hbar / 2)}{(\hbar-\psi)} \\
& \quad=-\frac{1}{2} \int_{\overline{\operatorname{Adm}}\left(g \stackrel{2}{\longrightarrow} 0,\left(t_{1}, t_{2}, \ldots, t_{2 g+2}\right)\right)}(-1)^{g} \psi^{2 g-1}=(-)^{g+1} \frac{1}{2} P_{2}(g) .
\end{aligned}
$$

So, altogether, the integral computed with Linearization B is

$$
I_{2}^{B}(g)=-\frac{1}{2}(2 g+1) L_{2}(g)-\sum_{i=0}^{g-1}(-1)^{g-i}\left(\begin{array}{c}
2 g+1 \\
2 i
\end{array}\right) P_{2}(g-i) L(i),
$$

where we have incorporated the last contribution in the summation by defining $L(0)=$ $1 / 2$.

Lemma 7 For any $i, P(i)=\frac{1}{2}$. 
Proof This follows easily from the fact that the $\psi$ classes that we are using are pulled back on the space of admissible covers from $\overline{\mathcal{M}}_{0,2 g+2}$ via an étale, degree $1 / 2$ map (this accounts for the hyperelliptic involution upstairs). The projective coarse moduli space of $\overline{\mathcal{M}}_{0,2 g+2}$ is $\bar{M}_{0,2 g+2}$, and the two spaces are birational. It is a classical result that the integral of $\psi$ to the top power on $\bar{M}_{0,2 g+2}$ is one, hence the lemma.

We can now equate the results obtained with the two different linearizations, to obtain a recursive formula for the $L_{2}(g)$ 's.

$$
-\frac{1}{2} L_{2}(g)=-\frac{1}{2}(2 g+1) L_{2}(g)-\sum_{i=0}^{g-1}(-1)^{g-i}\left(\begin{array}{c}
2 g+1 \\
2 i
\end{array}\right) P_{2}(g-i) L_{2}(i)
$$

After a tiny bit of elementary arithmetic we obtain

$$
L_{2}(g)=\frac{1}{2 g} \sum_{i=0}^{g-1}(-)^{g-i+1}\left(\begin{array}{c}
2 g+1 \\
2 i
\end{array}\right) L_{2}(i)
$$

\subsection{The generating function}

We now want to use relation (6) to compute the generating function:

$$
\mathcal{L}_{2}(x)=\sum_{i=0}^{\infty}\left(\frac{L_{2}(i)}{2 i+1 !}\right) x^{2 i+1} .
$$

Let us first of all differentiate this function,

$$
\frac{d}{d x} \mathcal{L}_{2}(x)=\sum_{i=0}^{\infty}\left(\frac{L_{2}(i)}{2 i !}\right) x^{2 i}
$$

Now let us compute

$$
\begin{aligned}
\frac{d}{d x} \mathcal{L}_{2}(x) \cdot \sin (x) & =\sum_{g=0}^{\infty} x^{2 g+1} \sum_{i=0}^{g}(-)^{g-i+1} \frac{L_{2}(i)}{2 i !(2 g-2 i+1) !} \\
& =\sum_{g=0}^{\infty} x^{2 g+1} \sum_{i=0}^{g}(-)^{g-i+1}\left(\begin{array}{c}
2 g+1 \\
2 i
\end{array}\right) \frac{L_{2}(i)}{2 g+1 !} \\
& =\sum_{g=0}^{\infty} x^{2 g+1}\left(\frac{L_{2}(g)}{2 g+1 !}\right) \\
& =\mathcal{L}_{2}(x) .
\end{aligned}
$$


Hence relation (6) translates to the following ODE on the generating function $\mathcal{L}_{2}(x)$ :

$$
\mathcal{L}_{2}^{\prime}(x) \cdot \sin (x)=\mathcal{L}_{2}(x), \quad \mathcal{L}_{2}(0)=0 .
$$

This equation integrates to give us $\mathcal{L}_{2}(x)=\tan (x / 2)$. Finally, recalling (5) we can conclude

$$
\mathcal{I}_{2}(x)=-\frac{1}{2} \tan \left(\frac{x}{2}\right)
$$

\subsection{A corollary}

Using result (8) it's now easy to compute the generating function for the second class of integrals we are interested in. Consider

$$
J_{2}(g)=\int_{\overline{\operatorname{Adm}}\left(g \stackrel{2}{\longrightarrow} \mathbb{P}_{\mathbb{C}}^{1},\left(t_{1}, t_{2}, \ldots, t_{2 g+2}\right)\right)} c_{2 g+2}\left(R^{1} \pi_{*} f^{*}\left(\mathcal{O}_{\mathbb{P}^{1}}(-1) \oplus \mathcal{O}_{\mathbb{P}^{1}}(-1)\right)\right),
$$

and the corresponding generating function

$$
\mathcal{J}_{2}(x)=\sum_{g=0}^{\infty}\left(\frac{J_{2}(g)}{2 g+2 !}\right) x^{2 g+2} .
$$

Again, there is a particularly favorable choice of linearizations:

\begin{tabular}{|l||c|c|}
\hline weight & over 0 & over $\infty$ \\
\hline \hline $\mathcal{O}_{\mathbb{P}^{1}}(-1)$ & -1 & 0 \\
\hline $\mathcal{O}_{\mathbb{P}^{1}}(-1)$ & 0 & 1 \\
\hline
\end{tabular}

The only contributing fixed loci must have valence 1 both over 0 and $\infty$. These are precisely the loci $F_{g_{1}, g_{2}}$ studied above. The explicit computation of the integral is

$$
\begin{aligned}
& \text { - } \quad x:(2 g+2) \int \frac{\lambda_{g} \Lambda_{g}(1)\left(\frac{\hbar}{2}\right)\left(-\frac{\hbar}{2}\right)}{\hbar(\hbar-\psi)(-\hbar)}=\frac{1}{4}(2 g+2) L_{2}(g) \\
& \stackrel{g_{1}}{\bullet} \quad g_{2}: 2\left(\begin{array}{c}
2 g+2 \\
2 g_{1}+1
\end{array}\right) \int \frac{\lambda_{g_{1}} \Lambda_{g_{1}}(1)\left(\frac{\hbar}{2}\right)}{\hbar(\hbar-\psi)} \int \frac{\lambda_{g_{2}} \Lambda_{g_{2}}(-1)\left(-\frac{\hbar}{2}\right)}{-\hbar(-\hbar-\psi)} \\
& =\frac{1}{2}\left(\begin{array}{c}
2 g+2 \\
2 g_{1}+1
\end{array}\right) L_{2}\left(g_{1}\right) L_{2}\left(g_{2}\right) \\
& \text { - : }(2 g+2) \int \frac{\lambda_{g} \Lambda_{g}(-1)\left(\frac{\hbar}{2}\right)\left(-\frac{\hbar}{2}\right)}{\hbar(-\hbar-\psi)(-\hbar)}=\frac{1}{4}(2 g+2) L_{2}(g)
\end{aligned}
$$


All previous integrals are computed over the appropriate unparmetrized admissible cover spaces. Adding everything together we obtain the relation

$$
J(g)=\frac{1}{2} \sum_{0}^{g}\left(\begin{array}{c}
2 g+2 \\
2 i+1
\end{array}\right) L_{2}(i) L_{2}(g-i) .
$$

(Recalling that we have defined $L_{2}(0)=1 / 2$.)

This relation allows us to obtain the generating function $\mathcal{J}_{2}(x)$. For this purpose it suffices to notice

$$
\mathcal{J}_{2}(x)=2 \mathcal{I}_{2}(x)^{2}=\frac{1}{2} \tan ^{2}\left(\frac{x}{2}\right)
$$

\section{Degree 3}

In this section we will compute the integral

$$
I_{3}(g):=\int_{\overline{\operatorname{Adm}}\left(g \stackrel{3}{\longrightarrow} \mathbb{P}_{\mathbb{C}}^{1},\left((3), t_{1}, \ldots, t_{2 g+2}\right)\right)} \operatorname{ev}_{(3)}^{*}(\infty) \cap c_{2 g+2}\left(R^{1} \pi_{*} f^{*}\left(\mathcal{O}_{\mathbb{P}^{1}} \oplus \mathcal{O}_{\mathbb{P}^{1}}(-1)\right)\right),
$$

for all genera $g$, and present the result in generating function form

$$
\mathcal{I}_{3}(x):=\sum_{g=0}^{\infty} \frac{I_{3}(g)}{2 g+2 !} x^{2 g+2} .
$$

\subsection{The strategy}

We will use localization to compute our integral. First of all, we choose an extremely convenient choice of linearizations on the $\mathbb{P}^{1}$-bundles $\mathcal{O}_{\mathbb{P}^{1}}$ and $\mathcal{O}_{\mathbb{P}^{1}}(-1)$. This will express our integral in terms of a Hodge integral over only one boundary component of the moduli space.

We then will introduce an auxiliary integral, that we know to vanish for elementary dimension considerations. Evaluating this integral via localization will produce relations between the integrals $I_{3}(g)$, for different genera $g$, integrals in degree 2 and simple Hurwitz numbers.

We are able to transform these relations into a linear differential equation for the generating function $\mathcal{I}_{3}(x)$. Finally, solving the ODE with the appropriate boundary conditions gives us the result. 


\subsection{The localization set-up}

We choose to linearize the bundle as in linearization $\mathrm{A}$ in the previous section:

\begin{tabular}{|l||c|c|}
\hline weight & over 0 & over $\infty$ \\
\hline \hline $\mathcal{O}_{\mathbb{P}^{1}}(-1)$ & -1 & 0 \\
\hline $\mathcal{O}_{\mathbb{P}^{1}}$ & 0 & 0 \\
\hline
\end{tabular}

For completely analogous reasons to the degree two case (see Figure 3.2), there is only one fixed locus, $F_{g}$, , contributing to the localization integral, consisting in a cover of $\mathbb{P}^{1}$ fully ramified over 0 and $\infty$, and a genus $g$ curve mapping with degree 3 to an unparametrized $\mathbb{P}^{1}$ sprouting from the point 0 .

The integral then becomes

$$
\begin{aligned}
I_{3}(g) & =\int_{F_{g, .}} \frac{\lambda_{g} \Lambda_{g}(1) \frac{2}{9} \hbar^{2}}{\hbar\left(\hbar-\psi_{3}\right)} \\
& =\frac{2}{9} \int_{\overline{\operatorname{Adm}}\left(g \stackrel{3}{\longrightarrow} 0,\left((3), t_{1}, \ldots, t_{2 g+2}\right)\right)} \lambda_{g} \lambda_{g-1} \psi_{3}+\lambda_{g} \lambda_{g-2} \psi_{3}^{2}+\cdots+\lambda_{g} \psi_{3}^{g} .
\end{aligned}
$$

With the sole purpose of keeping track of coefficients in a more natural way in what follows, we give a name to the rightmost integral without the $\frac{2}{9}$ in front of it:

$$
L_{3}(g):=\int_{\overline{\operatorname{Adm}}\left(g \stackrel{3}{\longrightarrow} 0,\left((3), t_{1}, \ldots, t_{2 g+2}\right)\right)} \lambda_{g} \lambda_{g-1} \psi_{3}+\lambda_{g} \lambda_{g-2} \psi_{3}^{2}+\cdots+\lambda_{g} \psi_{3}^{g} .
$$

\subsection{The auxiliary integral}

Let us now consider the following equivariant integral:

$$
\int_{\overline{\operatorname{Adm}}\left(g \stackrel{3}{\longrightarrow} \mathbb{P}_{\mathbb{C}}^{1},\left(t_{1}, \ldots, t_{2 g+4}\right)\right)} \mathrm{ev}_{1}^{*}(\infty) \cap c_{2 g+2}\left(R^{1} \pi_{*} f^{*}\left(\mathcal{O}_{\mathbb{P}^{1}} \oplus \mathcal{O}_{\mathbb{P}^{1}}(-1)\right)\right) .
$$

This integral must vanish for dimension reasons. Let us now evaluate this integral via localization. We now choose different linearizations for the two bundles, as indicated in the following table.

\begin{tabular}{|l||c|c|}
\hline weight & over 0 & over $\infty$ \\
\hline \hline $\mathcal{O}_{\mathbb{P}^{1}}(-1)$ & -1 & 0 \\
\hline $\mathcal{O}_{\mathbb{P}^{1}}$ & 1 & 1 \\
\hline
\end{tabular}


With this choice of linearizations, the explicit evaluation of the integral follows. We will again be invoking a famous relation by Mumford [11]:

$$
\Lambda_{g}(-1) \Lambda_{g}(1)=(-)^{g} \hbar^{2 g}
$$

$F_{g, 0} \quad \stackrel{g}{\bullet} \quad 3 \quad 0$

$$
\begin{array}{r}
3\left(\begin{array}{l}
2 g+3 \\
2 g+2
\end{array}\right) \int_{\overline{\operatorname{Adm}}\left(g \stackrel{3}{\longrightarrow} 0,\left((3), t_{1}, \ldots, t_{2 g+2}\right)\right)} \frac{(-)^{g} \hbar^{2 g}}{\hbar\left(\hbar-\psi_{3}\right)} \int_{\overline{\operatorname{Adm}}\left(0 \stackrel{3}{\longrightarrow} 0,\left((3), t_{1}, t_{2}\right)\right)}^{\frac{1}{-\hbar-\psi_{3}}\left(\frac{2}{9} \hbar^{2}\right)} \\
=(-)^{g+1} \frac{2}{3}\left(\begin{array}{l}
2 g+3 \\
2 g+2
\end{array}\right) \frac{1}{\hbar} \int_{\overline{\operatorname{Adm}}\left(g \stackrel{3}{\longrightarrow} 0,\left((3), t_{1}, \ldots, t_{2 g}+2\right)\right)} \psi^{2 g} \int_{\overline{\operatorname{Adm}}\left(0 \stackrel{3}{\longrightarrow} 0,\left((3), t_{1}, t_{2}\right)\right)} 1 \\
=(-)^{g+1} \frac{2}{3}\left(\begin{array}{l}
2 g+3 \\
2 g+2
\end{array}\right) P_{3,(3)}(g) L_{3}(0) \frac{1}{\hbar} .
\end{array}
$$

$F_{g_{1}, g_{2}} \quad \stackrel{g_{1}}{\bullet} \quad 3 \quad g_{2}$

$$
\begin{gathered}
3\left(\begin{array}{c}
2 g+3 \\
2 g_{1}+2
\end{array}\right) \int_{\overline{\operatorname{Adm}}\left(g_{1} \stackrel{3}{\longrightarrow} 0,\left((3), t_{1}, \ldots, t_{\left.\left.2 g_{1}+2\right)\right)} \frac{(-)^{g_{1}} \hbar^{2 g_{1}}}{\hbar\left(\hbar-\psi_{3}\right)}\right.\right.} \int_{\overline{\operatorname{Adm}\left(g_{2}\right.} \stackrel{3}{\longrightarrow} 0,\left((3), t_{1}, \ldots, t_{\left.\left.2 g_{2}+2\right)\right)}\right.}^{\frac{\lambda_{g_{2}} \Lambda_{g_{2}}(-1)}{-\hbar-\psi_{3}}\left(\frac{2}{9} \hbar^{2}\right)} \\
=(-)^{g_{1}+1} \frac{2}{3}\left(\begin{array}{c}
2 g+3 \\
2 g_{1}+2
\end{array}\right) \frac{1}{\hbar} \int_{\overline{\operatorname{Adm}}\left(g_{1} \stackrel{3}{\longrightarrow} 0,\left((3), t_{1}, \ldots, t_{2 g_{1}+2}\right)\right)} \psi^{2 g_{1}} \\
\int_{\overline{\operatorname{Adm}}\left(g_{2} \stackrel{3}{\longrightarrow} 0,\left((3), t_{1}, \ldots, t_{2 g_{2}+2}\right)\right)} \lambda_{g_{2} \lambda_{g_{2}-1} \psi_{3}+\cdots+\lambda_{g_{2}} \psi_{3}^{g_{2}}}=(-)^{g_{1}+1} \frac{2}{3}\left(\begin{array}{c}
2 g+3 \\
2 g_{1}+2
\end{array}\right) P_{3,(3)}\left(g_{1}\right) L_{3}\left(g_{2}\right) \frac{1}{\hbar} .
\end{gathered}
$$




$$
\begin{aligned}
& F_{0, g} \quad \stackrel{0}{\bullet} \quad 3 \quad \stackrel{g}{\bullet} \\
& 3\left(\begin{array}{c}
2 g+3 \\
2
\end{array}\right) \int_{\overline{\operatorname{Adm}}\left(0 \stackrel{3}{\longrightarrow} 0,\left((3), t_{1}, t_{2}\right)\right)} \frac{1}{\hbar\left(\hbar-\psi_{3}\right)} \\
& \int_{\overline{\operatorname{Adm}}\left(g \stackrel{3}{\longrightarrow} 0,\left((3), t_{1}, \ldots, t_{2 g}+2\right)\right)} \frac{\lambda_{g} \Lambda_{g}(-1)}{-\hbar-\psi_{3}}\left(\frac{2}{9} \hbar^{2}\right) \\
& =(-)^{g+1} \frac{2}{3}\left(\begin{array}{c}
2 g+3 \\
2
\end{array}\right) \frac{1}{\hbar} \int_{\overline{\mathrm{Adm}}\left(0 \stackrel{3}{\longrightarrow} 0,\left((3), t_{1}, t_{2}\right)\right)} 1 \\
& \int_{\overline{\operatorname{Adm}}\left(g \stackrel{3}{\longrightarrow} 0,\left((3), t_{1}, \ldots, t_{2 g+2}\right)\right)} \lambda_{g} \lambda_{g-1} \psi_{3}+\cdots+\lambda_{g} \psi_{3}^{g} \\
& =-\frac{2}{3}\left(\begin{array}{c}
2 g+3 \\
2
\end{array}\right) P_{3,(3)}(0) L_{3}(g) \frac{1}{\hbar} .
\end{aligned}
$$

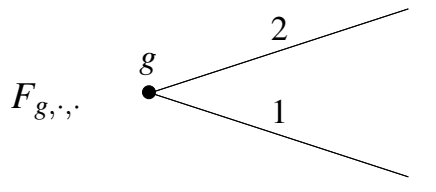

$$
\begin{aligned}
\left(\begin{array}{l}
2 g+3 \\
2 g+3
\end{array}\right) \int_{\overline{\mathrm{Adm}}\left(g \stackrel{3}{\longrightarrow} 0,\left(t_{1}, \ldots, t_{2 g+4}\right)\right)} \frac{(-)^{g} \hbar^{2 g}}{\hbar\left(\hbar-\psi_{t}\right)}\left(\frac{1}{2} \hbar^{2}\right) \\
=(-)^{g} \frac{1}{2} \frac{1}{\hbar} \int_{\overline{\operatorname{Adm}}\left(g \stackrel{3}{\longrightarrow} 0,\left(t_{1}, \ldots, t_{2 g+4}\right)\right)} \psi_{t}^{2 g+1}=(-)^{g} \frac{1}{2} P_{3,(t)}(g) \frac{1}{\hbar}
\end{aligned}
$$

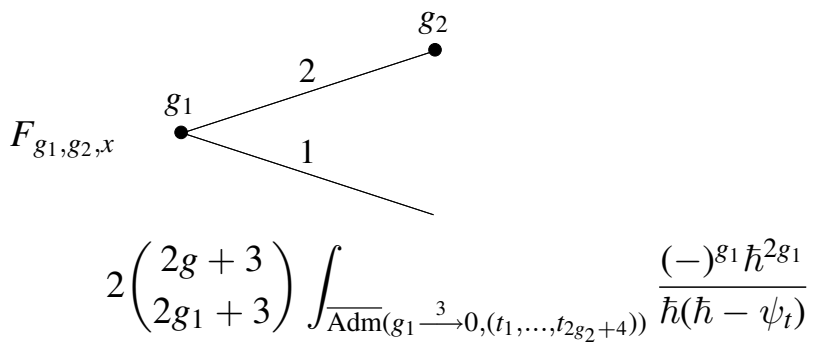

$$
\begin{aligned}
& \int_{\overline{\operatorname{Adm}}\left(g_{2} \stackrel{2}{\longrightarrow} 0,\left(t_{1}, \ldots, t_{2 g_{2}+2}\right)\right)} \frac{\lambda_{g_{2}} \Lambda_{g_{2}}(-1)}{-\hbar-\psi_{t}}\left(\frac{\hbar^{2}}{2}\right) \\
& =(-)^{g_{1}}\left(\begin{array}{c}
2 g+3 \\
2 g_{1}+3
\end{array}\right) \frac{1}{\hbar} \int_{\overline{\operatorname{Adm}}\left(g_{1} \stackrel{3}{\longrightarrow} 0,\left(t_{1}, \ldots, t_{\left.2 g_{2}+4\right)}\right)\right.} \psi_{t}^{2 g_{1}+1} \\
& \int_{\overline{\operatorname{Adm}}\left(g_{2} \stackrel{2}{\longrightarrow} 0,\left(t_{1}, \ldots, t_{2 g_{2}+2}\right)\right)} \lambda_{g_{2}} \lambda_{g_{2}-1}+\cdots+\lambda_{g_{2}} \psi_{t}^{g-1} \\
& =(-)^{g_{1}}\left(\begin{array}{c}
2 g+3 \\
2 g_{1}+3
\end{array}\right) P_{3,(t)}\left(g_{1}\right) L_{2}\left(g_{2}\right) \frac{1}{\hbar} \text {. }
\end{aligned}
$$




$$
\begin{aligned}
& F_{0, g, \cdot} \stackrel{2}{\bullet_{1}^{g}} \\
& 2\left(\begin{array}{c}
2 g+3 \\
3
\end{array}\right) \int_{\overline{\operatorname{Adm}}\left(0 \stackrel{3}{\longrightarrow} 0,\left(t_{1}, \ldots, t_{4}\right)\right)} \frac{1}{\hbar\left(\hbar-\psi_{t}\right)} \int_{\overline{\operatorname{Adm}}\left(g \stackrel{2}{\longrightarrow} 0,\left(t_{1}, \ldots, t_{2 g+2}\right)\right)} \frac{\lambda_{g} \Lambda_{g}(-1)}{-\hbar-\psi_{t}}\left(\frac{1}{2} \hbar^{2}\right) \\
& =\left(\begin{array}{c}
2 g+3 \\
3
\end{array}\right) \frac{1}{\hbar} \int_{\overline{\mathrm{Adm}}\left(0 \stackrel{3}{\longrightarrow} 0,\left(t_{1}, \ldots, t_{4}\right)\right)} \psi_{t} \int_{\overline{\mathrm{Adm}}\left(g \stackrel{2}{\longrightarrow} 0,\left(t_{1}, \ldots, t_{2 g+2}\right)\right)} \lambda_{g} \lambda_{g-1}+\cdots+\lambda_{g} \psi_{t}^{g-1} \\
& =\left(\begin{array}{c}
2 g+3 \\
3
\end{array}\right) P_{3,(t)}(0) L_{2}(g) \frac{1}{\hbar} .
\end{aligned}
$$

Finally, adding everything up, we obtain the following relation:

$$
\begin{aligned}
0=\frac{2}{3} \sum_{i=0}^{g}\left(\begin{array}{c}
2 g+3 \\
2 i+1
\end{array}\right)(-)^{g-i+1} P_{3,(3)}(g-i) L_{3}(i) & \\
& +\sum_{i=0}^{g}\left(\begin{array}{c}
2 g+3 \\
2 i
\end{array}\right)(-)^{g-i} P_{3,(t)}(g-i) L_{2}(i)
\end{aligned}
$$

\subsection{The generating function}

Now for the less deep but more delicate part of our computation: we need to extract from relation (11) a differential equation involving our desired generating function.

Let's start with a preliminary lemma:

Lemma 8 For all $g \geq 0$,

(1) $P_{3,(3)}(g)=3^{2 g}$.

(2) $P_{3,(t)}(g)=\left(3^{2 g+2}-1\right) / 2$.

Proof (1) Consider the map

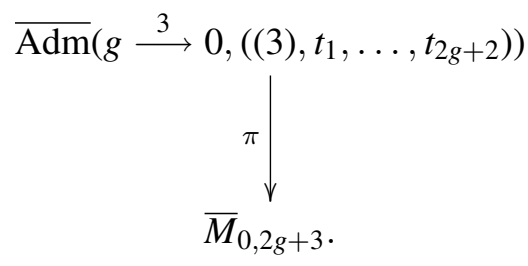


It's a classical result that

$$
\int_{\bar{M}_{0,2 g+3}} \psi_{1}^{2 g}=1
$$

Since our psi class is just the pull-back of $\psi_{1}$ on $\overline{\mathcal{M}}_{0,2 g+3}$, and this space is birational to its projective coarse moduli space $\bar{M}_{0,2 g+3}$, our lemma is proven if we show that $\pi$ has degree $3^{2 g}$. This is a classic Hurwitz number, counting the number of degree 3 covers of the Riemann sphere with a triple ramification point and simple ramification otherwise.

The problem is purely combinatorial. We are free to choose a three-cycle in $S_{3}$ giving the monodromy of the triple point. The triple point automatically guarantees that our cover is connected. Then we are free to choose cycles for the first $(2 g+1)$ simple ramification points. The monodromy of the last ramification point is determined by the fact that the product of all monodromies should be the identity. So alltogether we had a choice of $2 \cdot 3^{2 g+1}$ elements of $S_{3}$. We now need to divide by the conjugation action of $S_{3}$ on itself, that geometrically amounts to simply relabelling the sheets of the cover. Finally we obtain the desired $3^{2 g}$ non isomorphic covers.

(2) Similarly, we need to count the number of degree 3 covers of $\mathbb{P}^{1}$ with $2 g+4$ simple ramification points. Paralleling the previous argument, we can choose $(2 g+3)$ cycles freely. But we have to beware of disconnected covers. These can happen only if we chose always the same cycle. So in total we have $3^{2 g+3}-3$ choices. Dividing now by 6 we obtain our claim.

Let us now translate relation (11) in the language of generating functions. Define

$$
\begin{aligned}
\mathcal{L}_{3}(x) & :=\sum_{g=0}^{\infty} \frac{L_{3}(g)}{2 g+2 !} x^{2 g+2}, & \mathcal{L}_{2}(x) & :=\sum_{g=0}^{\infty} \frac{L_{2}(g)}{2 g+1 !} x^{2 g+1}, \\
\mathcal{P}_{3,(3)}(x) & :=\sum_{g=0}^{\infty}(-)^{g} \frac{P_{3,(3)}(g)}{2 g+2 !} x^{2 g+2}, & \mathcal{P}_{3,(t)}(x) & :=\sum_{g=0}^{\infty}(-)^{g} \frac{P_{3,(t)}(g)}{2 g+3 !} x^{2 g+3} .
\end{aligned}
$$

Then our relation (11) becomes an ordinary differential equation on the generating functions:

$$
\frac{2}{3} \mathcal{P}_{3,(3)} \mathcal{L}_{3}^{\prime}-\mathcal{P}_{3,(t)} \mathcal{L}_{2}^{\prime}=0
$$

By Lemma 8 we can explicitly describe the Hurwitz numbers' generating functions

$$
\mathcal{P}_{3,(3)}(x)=\frac{1-\cos (3 x)}{9} ; \quad \mathcal{P}_{3,(t)}(x)=\frac{3 \sin (x)-\sin (3 x)}{6} .
$$


Also, we do know the generating function for the degree 2 theory, hence

$$
\mathcal{L}_{2}^{\prime}(x)=\frac{d}{d x} \tan \left(\frac{x}{2}\right)=\frac{1}{2 \cos ^{2}\left(\frac{x}{2}\right)} .
$$

Finally, we have reduced our problem to integrating the following:

$$
\begin{aligned}
& \tilde{\mathcal{L}}_{3}^{\prime}(x)=\frac{9}{8} \frac{3 \sin (x)-\sin (3 x)}{(1-\cos (3 x)) \cos ^{2}\left(\frac{x}{2}\right)}, \\
& \tilde{\mathcal{L}}_{3}(0)=0 .
\end{aligned}
$$

This ODE integrates to

$$
\mathcal{L}_{3}(x)=\frac{9}{2}\left(\frac{1}{4 \cos ^{2}\left(\frac{x}{2}\right)-1}-\frac{1}{3}\right) .
$$

Now let us remember that the generating function $\mathcal{I}_{3}(x)$ is smply $(2 / 9) \mathcal{L}_{3}(x)$. After just a little bit of trigonometry clean-up we obtain:

$$
\mathcal{I}_{3}(x)=\frac{4}{3} \frac{\sin ^{3}\left(\frac{x}{2}\right)}{\sin \left(\frac{3 x}{2}\right)}
$$

\subsection{A corollary}

In a completely similar fashion to degree 2 , it is possible to obtain from the previous computation the generating function for the integrals:

$$
J_{3}(g)=\int_{\overline{\operatorname{Adm}}\left(g \stackrel{3}{\longrightarrow} \mathbb{P}_{\mathbb{C}}^{1},\left(t_{1}, \ldots, t_{2 g+4}\right)\right)} c_{2 g+4}\left(R^{1} \pi_{*} f^{*}\left(\mathcal{O}_{\mathbb{P}^{1}}(-1) \oplus \mathcal{O}_{\mathbb{P}^{1}}(-1)\right)\right) .
$$

The answer is

$$
\mathcal{J}_{3}(x)=\sum_{g=0}^{\infty}\left(\frac{J_{3}(g)}{2 g+4 !}\right) x^{2 g+4}=3 \mathcal{I}(x)^{2}=\frac{16}{3} \frac{\sin ^{6}\left(\frac{x}{2}\right)}{\sin ^{2}\left(\frac{3 x}{2}\right)} .
$$

\section{References}

[1] D Abramovich, A Corti, A Vistoli, Twisted bundles and admissible covers, Comm. Algebra 31 (2003) 3547-3618 MR2007376

[2] M F Atiyah, R Bott, The moment map and equivariant cohomology, Topology 23 (1984) 1-28 MR721448

[3] J Bryan, R Pandharipande, The local Gromov-Witten theory of curves arXiv: math.AG/0411037 
[4] T Ekedahl, S Lando, M Shapiro, A Vainshtein, On Hurwitz numbers and Hodge integrals, C. R. Acad. Sci. Paris Sér. I Math. 328 (1999) 1175-1180 MR1701381

[5] T Ekedahl, S Lando, M Shapiro, A Vainshtein, Hurwitz numbers and intersections on moduli spaces of curves, Invent. Math. 146 (2001) 297-327 MR1864018

[6] C Faber, R Pandharipande, Hodge integrals and Gromov-Witten theory, Invent. Math. 139 (2000) 173-199 MR1728879

[7] I P Goulden, D M Jackson, R Vakil, Towards the geometry of double Hurwitz numbers, Adv. Math. 198 (2005) 43-92

[8] T Graber, R Vakil, Hodge integrals and Hurwitz numbers via virtual localization, Compositio Math. 135 (2003) 25-36 MR1955162

[9] J Harris, D Mumford, On the Kodaira dimension of the moduli space of curves, Invent. Math. 67 (1982) 23-88 MR664324

[10] E Ionel, Topological recursive relations in $H^{2 g}\left(M_{g, n}\right)$, Invent. Math. 148 (2002) 627-658 MR1908062

[11] D Mumford, Towards an enumerative geometry of the moduli space of curves, from: “Arithmetic and geometry, Vol. II”, Progr. Math. 36, Birkhäuser, Boston (1983) 271-328 MR717614

Department of Mathematics, University of Utah

155 South 1400 East, Salt Lake City UT 84112, USA

Department of Mathematics, University of Michigan

2074 East Hall, 530 Church Street, Ann Arbor MI 48109-1043, USA

crenzo@umich.edu, renzo@math.utah.edu

Received: 9 March 2005

Geometry \& Topology Monographs 8 (2006) 